\title{
Les fondements littéraires de la personne
}

\author{
Jonathan Wenger
}

\section{OpenEdition}

Journals

Édition électronique

URL : https://journals.openedition.org/edl/1847

DOI : $10.4000 /$ edl. 1847

ISSN : 2296-5084

\section{Éditeur}

Université de Lausanne

\section{Édition imprimée}

Date de publication : 15 décembre 2019

Pagination : 31-48

ISBN : 978-2-940331-72-7

ISSN : 0014-2026

\section{Référence électronique}

Jonathan Wenger, «Les fondements littéraires de la personne », Études de lettres [En ligne], 311 | 2019, mis en ligne le 15 décembre 2021, consulté le 16 décembre 2021. URL : http://

journals.openedition.org/edl/1847 ; DOI : https://doi.org/10.4000/edl.1847

Ce document a été généré automatiquement le 16 décembre 2021.

(c) Études de lettres 


\title{
Les fondements littéraires de la personne
}

\author{
Jonathan Wenger
}

1 La recherche consacrée à l'œuvre de Denis de Rougemont néglige souvent deux éléments pourtant fondamentaux: sa composante religieuse et métaphysique d'une part et de l'autre, sa dimension littéraire ${ }^{1}$.

2 Si sa foi protestante se perçoit clairement dans ses écrits, elle est généralement reléguée au statut de croyance personnelle, sans égard pour le rôle axiomatique qu'elle joue dans ses théories personnalistes, et par extension fédéralistes ${ }^{2}$. Le cadre qui nous est imparti ne nous permet d'en dire que l'indispensable, tant cette foi est liée à sa pensée philosophique et littéraire ${ }^{3}$.

3 La présence de la littérature dans son œuvre est encore moins considérée. Or, de nombreux éléments nous incitent à croire que la littérature avait pour lui une importance d'un autre ordre ; en rappelant les fondements généraux du personnalisme, puis en détaillant deux métaphores essentielles (la dramaturgie de l'existence et les vertus de l'écrivain) que Rougemont développe dans sa pensée $e^{4}$ nous espérons mettre en évidence non seulement la place qu'y occupe la littérature, mais encore son rôle fondateur ${ }^{5}$.

\section{Éléments de personnalisme}

4 En 1930, Rougemont monte à Paris comme directeur littéraire des Éditions «Je Sers ». L'année suivante, il y rencontre Alexandre Marc et s'implique dans les divers groupements personnalistes, notamment Plan, Esprit et L'Ordre Nouveau. L'objectif de ces divers groupes peut se résumer par la volonté d'élaborer une nouvelle pensée de l'ordre social, à une époque où celui-ci leur apparaissait compromis. Du point de vue politique, leur crainte se focalisait sur la prédominance de deux tendances : l'individualisme, où les rapports humains s'atrophient dans un souci excessif de soi ; et le fascisme, où les individus se dissolvent dans l'État. La mise au point d'une troisième voie, où chaque 
citoyen serait co-responsable de la Cité, se retrouvera chez Rougemont dans sa théorie du fédéralisme.

5 Quant à énoncer des fondements théoriques, Rougemont ne s'y est risqué qu'une seule fois, dans le texte "Définition de la personne». Les dizaines de textes de sa main abordant la question se consacrent le plus souvent à un aspect spécifique : la personne citoyenne, religieuse, culturelle, etc., selon le cadre (colloque ou conférence) où il représentait ces mouvements. En recoupant l'ensemble des éléments évoqués dans ces différentes circonstances pour esquisser une définition synthétique, on constate que l'articulation de quatre concepts-clés donne un résultat simple : une personne, c'est un individu en tant qu'il agit selon sa vocation. Précisons que cette action doit se dérouler au sein d'une communauté.

6 La vocation n'est rien moins que la mission personnelle que Dieu attribue à chacun dans son existence ${ }^{6}$. L'individu n'est rien en soi. Il n'est, comme l'étymologie l'indique, qu'un élément divisé, considéré à part au sein de la communauté humaine, voire de l'existence. Mais quand il agit selon sa vocation, sa nécessité propre, qu'il la réalise (où « réaliser » est à prendre au sens premier « d'amener au réel », «d'incarner »), il est à ce moment une personne, c'est-à-dire un agent qui, en se réalisant, participe à réaliser le plan divin et se trouve de ce fait à la fois lié à la communauté humaine et apportant du sens au monde ${ }^{7}$ :

Voilà l'homme que j'appelle une personne: il est à la fois libre et engagé, et il est

libéré par cela même qui l'engage envers son prochain, je veux dire par sa

vocation ${ }^{8}$.

7 Outre ce schéma (dont on nous pardonnera la concision), un autre rappel est important: le combat contre le scientisme déterministe et la possibilité de l'action libre.

8 Rougemont s'est opposé avec virulence à une certaine conception du monde rationaliste, voire "ratiocentriste", qu'il fait remonter à Descartes. Il impute au philosophe l'introduction dans la pensée du primat absolu de la raison dans l'expérience. Effet pervers de cette révolution, notre compréhension de la réalité, jusque-là unie, se retrouve divisée ${ }^{9}$. En se servant de la seule raison pour appréhender le monde, nous avons perdu de vue la dimension active de l'existence, sa part proprement humaine : le fait que nous réalisons nos actes, et que nous nous réalisons nous-même par ce biais.

9 Tout acte, considéré du seul point de vue d'un observateur, ignore la dimension existentielle de celui qui l'accomplit: le recul du savant l'amène à dénigrer cette composante pour n'y voir que l'effet de déterminismes. La nature, comme l'existence, ne sont alors plus qu'un jeu de forces autonomes et réglées où, pour ainsi dire, ne se passent plus que des "choses qui arrivent ", sans une volonté pour les occasionner. On ne trouvera forcément pas plus de sens à l'échelle de la société : et l'histoire, comme la sociologie, deviennent des discours taxidermisant un monde réduit à l'immanence ${ }^{10}$ un discours scientifique ne pouvant rendre compte que de l'observable. Or, «l'acte est insaisissable parce qu'il est saisissant $»^{11}$, à la fois saisie et création de l'événement.

10 Rougemont soutient qu'il est impossible de déduire des lois expliquant le comportement humain, à moins que les humains à qui elles sont présentées ne les acceptent et ne les appliquent, c'est-à-dire s'y aliènent :

La plupart des « lois scientifiques » qu'on invoque à gauche et à droite pour justifier

les trahisons de la personne, n'existent réellement qu'à partir du moment où l'homme 
n'existe plus en tant que tel. Que l'homme s'affiche, qu'un seul homme s'affirme, et le pouvoir des lois diminue aussitôt. [...] Cette pensée sociologique [...] voudrait codifier la loi d'évolution des «masses " comme si les masses n'étaient pas faites d'hommes, c'est-à-dire d'éléments imprévisibles ${ }^{12}$.

11 Et si cette aliénation persiste, alors le récit de l'existence est simplement gouverné par ces règles. Bernard Groethuysen a merveilleusement résumé la perte du sens induite par la vision scientifique du monde :

Les sciences ont rompu l'unité de ce monde, en détruisant ce qu'on pourrait appeler le roman universel du christianisme, sans d'ailleurs, empressons-nous de le dire, lui substituer un autre roman. Elles disent, en quelque sorte : il n'y a pas de roman du tout. Toutes vos histoires sont également fausses, car il n'y a pas d'histoire. L'univers n'est pas un roman. Un récit n'est pas une explication. La logique du roman n'est pas celle des sciences. $\mathrm{Si}$, au cours d'un roman, un des personnages meurt, cela peut parfaitement être motivé par l'ensemble de l'œuvre, sans que l'auteur nous fournisse la moindre explication scientifique de la mort du personnage. Cette mort a un sens qui dérive de l'ensemble de l'œuvre. Il fallait que le personnage mourût; la composition du roman ne permettait pas d'autre issue. Ainsi de l'épopée chrétienne. Tout y est nécessaire, tout y est «logique », parce que tout s'interprète en fonction de l'œuvre, et que tout a un sens ${ }^{13}$.

Nous en arrivons ainsi à la dimension littéraire de ce personnalisme: celle de l'existence comme devant être à la fois action et narration.

\section{Une dramaturgie de l'existence}

13 Unique texte tentant une explication globale, "Définition de la personne " nous intéresse notamment pour être soutenu de bout en bout par une seule métaphore, strictement littéraire : le monde est un théâtre.

Sur la scène du monde, où nous avons été placés, dans ce drame qu'il nous faut jouer sans le connaître, c'est-à-dire qu'il nous faut inventer, il y a des figurants qui n'ont pas de visage; mais ceux qu'on voit sont les acteurs qui jouent leur rôle d'hommes et qui créent leur destin : ceux-là seuls sont les dramatis personae, ceux-là seuls sont présents, parce qu'ils représentent.

[...] Mais cela dit, il reste à savoir pourquoi tel figurant jeté dans une intrigue insaisissable devient tout à coup un acteur, et se met à se comporter tout comme s'il connaissait le fil du drame. D'où lui vient tout à coup l'assurance que ce qu'il fait est dans son rôle? Pour quelle raison sort-il du chœur des anonymes résignés, pour revêtir un vrai visage, un nom et une autorité, une attitude d'auteur de son propre destin? C'est ce que l'on ne voit point.

C'est ce que nul ne peut voir ni ne verra jamais, cependant que chacun peut voir qu'il existe, en fait, des personnes; cependant que chacun peut savoir en quoi consiste sa propre personne.

Ma personne, c'est ma présence au monde et à moi-même conjointement aux vrais objets, aux vrais humains, et à ma vraie responsabilité ${ }^{14}$.

Être justement présent au monde, c'est donc véritablement occuper un rôle :

La personne chrétienne, ce sera le rôle que Dieu attribue à chaque homme dans son plan. [...] Ainsi la dignité de chaque individu est garantie non pas du seul fait qu'il existe physiquement, mais du fait qu'il peut incarner une volonté particulière de Dieu ${ }^{15}$.

Cette image, il la veut « autorisée par l'étymologie du mot personne ${ }^{16}$, renvoyant au fameux per-sonare, "sonner au travers ». Elle expliquerait que le masque désignant le rôle de l'acteur dans le théâtre antique ait reçu le nom de persona. Outre qu'il permettait au spectateur d'identifier un caractère-type, le masque était également 
façonné de manière à amplifier la portée de la voix de l'acteur. Dans cette métaphore, ce masque désigne donc la personne par le rôle qui lui est assigné, comme par la fonction de faire retentir l'ordre de Dieu dans l'exécution de son rôle ${ }^{17}$.

La métaphore du rôle, et du masque qui ne dissimule pas, mais situe, est loin d'être gratuite et sans applications. Il l'a lui-même illustrée dans son unique pièce de théâtre, Nicolas de Flue. La structure dramaturgique y illustre le drame concret de toute personne - et plus spécialement de l'intellectuel, du «clerc $»^{18}$ ou de l'écrivain. Le premier acte montre le protagoniste se retirant de ses fonctions publiques, écœuré par la pusillanimité de ses semblables, puis quittant sa famille pour se rapprocher davantage de Dieu; le constat de l'insuffisance du monde, ainsi que la quête d'absolu, évoquent l'attention à sa vocation (voir infra, sur la notion d'" originalité »). Au deuxième acte advient la crise sociale, dont Nicolas est partiellement et indirectement responsable pour avoir abandonné l'exercice public de la justice (selon le reproche de démission que Rougemont adresse aux clercs tout au long de Penser avec les mains). Au troisième acte enfin, contre son premier désir de solitude, l'ermite, dont la réputation a incomparablement grandi, retourne à la Cité, reprend sa place d'autorité dans la vie commune et permet la résolution des conflits.

Cet oratorio lui a été commandé pour l'Exposition nationale de 1939. Rougemont en a profité pour y inscrire un plaidoyer sur l'engagement de la Suisse dans ces heures de crise - et n'a pas manqué de la composer de façon à illustrer son personnalisme ${ }^{19}$.

17 Il n'y a d'autres façons de comprendre les écrits de Rougemont sur la question que de présupposer une égalité entre un rôle encore à interpréter et une dramaturgie déjà écrite: mais l'actualisation doit avoir lieu, moins par prédestination que pour son inscription dans l'éternité. Le monde étant ainsi nécessairement, pour qui en saisira l'occasion, un récit doté de sens, on comprend que l'interprète de l'œuvre rougemontienne se trouve placé dans une perspective narrative correspondant autant à une vision providentielle qu'existentielle. Car sans acte libre et consenti, les éléments précités n'ont aucune valeur logique (ce qui importe peu), mais surtout aucun enjeu (ce qui importe réellement à notre auteur). Il s'agit maintenant de se demander comment opère un homme libre, coïncidant avec sa propre nécessité.

\section{Les vertus de l'écrivain, ou penser avec les mains}

En rédigeant Penser avec les mains, Rougemont entendait donner une approche complète du problème social que diagnostiquaient les personnalistes, ainsi qu'un programme de solution. La seconde partie du volume s'attache à établir ce qu'on pourrait appeler une nouvelle hygiène de vie, une rigoureuse éthique de la personne entière - et non une discipline intellectuelle.

vigueur morale requise se fonde sur la pratique de huit vertus. Les quatre premières concernent la personne dans son rapport au monde et à ses semblables : à savoir le réalisme, la violence ("qui n'est pas la brutalité », mais "fait violence à un état de chose $\left.\aleph^{20}\right)$, l'autorité et le risque. Quant aux quatre suivantes, elles se rapportent au contraire à la seule personne l'exerçant: il s'agit de l'originalité, de l'ascétisme de l'expression, de l'imagination et du style.

On voit bien que ces quatre dernières vertus ressemblent moins aux catégories d'un philosophe que d'un écrivain ${ }^{21}$; et leurs dénominations, pour ne pas recouvrir 
exactement les notions auxquelles nous sommes habitués, n'en découlent pas moins de la pratique littéraire de Rougemont. Elles nous permettront ainsi d'expliciter sa conception de l'écrivain.

21 L'originalité n'est pas le tour de prestidigitateur faisant apparaître une tournure ou un genre jusque-là inconnu, mais la faculté à se trouver en contact avec notre origine, soit notre vocation ${ }^{22}$. Et si Rougemont défend à la fois l'option nécessaire à laquelle nous devons correspondre et le choix libre (pour mettre encore une fois en avant cette antinomie spinoziste), on comprend que la nécessité et la créativité se retrouvent au même point.

L'ascétisme de l'expression reprend un thème développé dans plusieurs articles des années 1930. Contre un langage débordant à la fois de platitudes superflues (les médias) et d'idiomes sur-spécialisés (les « clercs » isolés en leur tour d'ivoire), toute possibilité de dialogue s'est perdue. Contre cette "décadence des lieux communs", conçus ici comme des points de repère et non comme des banalités, cette austérité linguistique doit permettre de retrouver un langage atteignant tout un chacun. Cette division dépasse le seul rôle de la communication: elle est également tributaire du reproche scientiste évoqué plus haut, nommé ici le " péché abstracteur »"23 qui a divisé le mot et la pensée, «l'être et l'expression »- ou, comme il le dit ailleurs, qui a occasionné «le divorce [...] entre la culture et l'action, entre le cerveau et la main $»^{24}$. Ainsi, «la question du langage revient à celle de l'exacte incarnation des idées dans les mots $»^{25}$. Cette ascèse, on le verra plus bas, est la condition du style. Précisons que dans un texte inédit en français, Rougemont a précisé les choses ainsi :

Un nouveau classicisme, un nouvel ascétisme du langage me paraît aujourd'hui d'une importance sociale incalculable : car c'est à la faveur du vague des mots, de l'excès des formules romantiques vulgarisées par les hauts-parleurs que les militaires ont pu répandre dans les masses des sophismes aussi meurtriers que celui de «l'espace vital» par exemple ${ }^{26}$.

L'imagination est également dotée d'une connotation principalement active: «L'étymologie grecque du mot idée, c'est le verbe voir. [...] L'imagination du langage naît d'une vision créatrice du monde $»^{27}$. Ainsi qu'il le développe ailleurs, « la vision est un acte $"$ :

Voir, c'est juger en même temps que former : - c'est transformer ${ }^{28}$.

Ainsi, imaginer ne veut pas dire recomposer passivement des formes à partir de schémas connus : c'est proprement créer une image et l'orienter avec l'œuvre :

c'est voir le but, c'est voir le tout, qu'il s'agit de rejoindre et d'accomplir. Voilà le vrai moteur de la pensée ${ }^{29}$.

Il le redira bien plus tard, à propos encore de l'écriture :

Quand je saurai pourquoi [j'écris], j'aurai fini d'écrire (idéalement). J'aurai touché à la fin de l'écriture, ou mieux, j'aurai rejoint ma fin, qui est de me former une pensée vécue dans l'écriture ${ }^{30}$.

Si l'imagination permet la vision et la création de l'œuvre, son incarnation, le style correspond à son action réunie :

Si le style est de l'homme même, on peut dire plus précisément qu'il est de l'action même, de la personne en exercice ${ }^{31}$.

Il précise cette pensée notamment à propos de Gide, notant la nécessité d'aller à l'encontre de la sincérité que prônait celui-ci; il ne devait pas en venir à supprimer "de ses carnets les pages qu'il jugeait trop “écrites". Entendons que l'effort de style y 
déformait la spontanéité, et se voit condamné comme insincère $»^{32}$. Le procédé est diabolisé chez Gide comme une intervention des conventions d'écriture arbitraires qui contraignent la vérité naturelle de l'individu (ce qui nous ramène au manque d'originalité comme d'ascétisme linguistique ${ }^{33}$. Or, pour Rougemont, il s'agit précisément d'aller contre un «moi » qui se restreindrait au naturel, se laissant ainsi aller à pousser comme un jardin de mauvaises herbes - autrement dit, tout l'inverse de la culture. Le style est l'effort de l'homme qui, au sens premier, se cultive, qui cherche à avoir un effet sur lui-même, pour aller à son but. Rougemont entend ainsi opposer à cette spontanéité « une sincérité qu'on pourrait nommer constructive », exigeant de la personne qu'elle ose affirmer "[ses] valeurs et de [ses] préférences », qui ne sont rien moins que :

[...] le style de l'existence. Il est en nous le trait révélateur d'une unité intentionnelle, d'un parti-pris aussi sincère, si ce n'est plus, que la pluralité des pulsions instinctives. Fixer, en les notant, certaines contractions d'humeurs, c'est parfois moins « se réciter » que se déformer ${ }^{34}$.

Mais si le style est révélateur de la personne, il l'est aussi de son acte, de son impact ${ }^{35}$ sur le monde ; autrement dit, de l'inscription littérale de son message. Il réalise, donne forme. Se demandant comment la théologie pouvait être liée à la littérature, comment espérer pratiquer les deux sans que l'une n'asservisse l'autre, il conclut que leur rapport ne doit pas être envisagé comme un discours où leurs expressions resteraient dissociées, alternant sans se mêler. Au contraire, elles doivent être unies de la même façon que le mot et la pensée; et cette réunion dans l'action, c'est le style au sens non plus élargi, mais strict du mot :

Ce qu'il importe de rappeler ici, c'est que toute œuvre littéraire, si profane qu'en soit le sujet, implique une théologie, (fût-ce à l'insu de son auteur), et qu'elle l'exprime par les mouvements mêmes du style, plus fidèlement et d'une manière plus contraignante que par son argumentation ${ }^{36}$.

Les vertus austères que préconisent Rougemont s'inscrivent dans sa perspective de l'action: rien ne sert d'écrire par vanité : le geste graphique est celui qui entend imprimer un sens, coup de poing sur la table ou signature décisive. Isoler la littérature dans un monde des idées serait encore céder au "péché abstracteur». Ce qui nous amène à une dernière question: l'écrivain est-il un exemple particulier de la personne?

\section{Les personnes du drame, ou l'écrivain et l'existence}

Qu'est-ce donc qu'une vocation réussie - donc, en dernière instance, qu'une existence réussie? Rougemont a consacré à la question Les personnes du drame. Le seul titre du volume est programmatique : en traduisant dramatis personae, expression par laquelle le scripteur d'une pièce de théâtre liste ses personnages, il lui ajoute la connotation existentielle qu'il confère à la notion de personne.

31 Les sujets du livre sont évoqués en sous-titre : Ramuz ; Claudel ; Gide ; Luther ; Goethe; Kafka; Kierkegaard. Significativement, Rougemont concentre donc la personne, le personnage et l'auteur dans une même matière. Rougemont y analyse plusieurs existences, symptomatiques par leur grandeur, où cette théâtralisation semble rappeler l'écho d'une vie vécue selon la vocation. Remarquons que Les personnes du drame examine différents écrivains non sur le seul plan biographique ou historique (à la façon 
classique de «sa vie, son œuvre »), ni sur le plan de l'interprétation textuelle, mais sur le plan qui réunit leur action (l'écriture) avec leur vie (le décor où l'action peut se dérouler), exprimée dans son espace propre et non l'espace positif. Ces lectures portent sur le plan de l'œuvre :

Car toute œuvre est le témoignage d'un drame entre l'homme et lui-même, elle est ce drame, rendu visible, et c'est dans le drame qu'existe la vérité totale d'un être ${ }^{37}$.

La notion de drame prend ici toute sa portée : les actes d'une personne deviennent, en se vouant à l'accomplissement de cette "volonté particulière de Dieu " $^{38}$, l'action du drame, son cours. Cela nous renseigne encore sur la façon dont Rougemont refuse la réduction à l'immanence. Il ne s'agit pas, en effet, de concevoir l'œuvre littéraire comme un élément à part de la vie de son auteur, un épiphénomène se situant à telle étape du cours de l'Histoire. L'œuvre, comme l'existence de la personne, est une trame sensée qui tend vers un but. Son orientation dans l'Histoire ne se conçoit pas selon un déroulement $d u$ temps en vue d'une fin perceptible à vue terrestre ${ }^{39}$, mais dans l'éternité d'une incarnation réussie. Sa vérité ne relève pas de la factualité historique, d'un calque du discours sur les faits, mais de la création d'un sens comparable à celui instauré par l'unité de la composition littéraire. Ce que Rougemont dit de l'homme à l'égard de l'incarnation ${ }^{40}$ vaut ainsi pour l'œuvre :

Il me semble que toute incarnation d'une pensée dans une vie ou d'une vocation dans un individu " figure » la synthèse en un seul être, en un seul acte, en une seule œuvre, de deux natures distinctes ou même contradictoires [...] Cette manière de saisir et de créer des relations par nul autre prévues, voilà précisément ce qu'on peut appeler le style "personnel » d'un auteur, ou d'ailleurs de n'importe quel homme responsable de son existence. Il s'agit là d'un phénomène qui déborde dès l'origine le fait d'écrire, au sens étroit ${ }^{41}$.

L'œuvre est un témoignage de son auteur - et par extension, du plan divin réalisant un monde faisant sens. Et c'est peut-être un défaut de lecteur que d'oublier qu'elle n'est pas d'abord faite pour être lue, mais pour être faite tout court, parce qu'elle est l'acte d'un auteur. Parlant une langue propre qui atteste de son originalitét ${ }^{42}$, elle témoigne d'une capacité à créer et à se créer. Elle s'oppose ainsi aux discours scientistes, qu'ils relèvent de l'histoire, de la psychologie ou de la sociologie, qui tous s'obstinent à ne rendre de l'être humain que sa dimension quantifiable. L'étude de l'homme et l'étude de l'œuvre sont à tel point synonymes qu'il peut conclure son introduction en disant que $«$ je n'ai pas fait de la critique dans cet ouvrage, mais des exercices spirituels ${ }^{43}$.

Cette remarque ne doit pas se lire comme une minorisation de la littérature. Tout au contraire, il s'agit de réajuster à deux titres la portée de celle-ci : au niveau spirituel, en la montrant comme un geste dicté par la vocation; et au niveau humain, en la montrant comme un acte dépassant la seule occupation des "gendelettres", pour s'exercer dans la réalité pleine de l'existence.

\section{Conclusion : l'écrivain, moraliste engagé}

Qui est donc, dans la pensée de Rougemont, l'auteur ou l'acteur? Quelques éléments d'une profession de foi, le discours prononcé lors de la remise du Grand Prix Schiller, nous renseignent à cet égard :

Depuis le $\mathrm{XIX}^{e}$ siècle romantique, le grand public et la plupart des critiques semblent penser que la littérature c'est poésie, roman, théâtre, et que création littéraire serait synonyme de fiction. 
Voilà qui est méconnaître à tout le moins l'histoire de la littérature française. Les chefs d'œuvre de notre langue, la floraison de son vocabulaire, la grande allure et les éclats du style, ne se voient guère chez les romanciers, à part Stendhal, ni même chez les poètes français, à part Baudelaire et Saint-John Perse. Mais dans Calvin, l'initiateur de la langue des idées en France, et dans Montaigne, inventeur des Essais précisément; puis dans le Pascal des Pensées, le Descartes du Discours, le Montesquieu des Lettres persanes, le Voltaire des écrits polémiques et pas du tout des tragédies en vers, le Rousseau des Rêveries et des Confessions, le Chateaubriand des Mémoires d'Outre-Tombe, le Victor Hugo des discours sur l'Europe et pour la Paix, le Rimbaud d'Une saison en enfer, et tout près de nous, le Valéry de Variété et de Tel Quel, l'André Breton des Manifestes surréalistes, le Saint-Exupéry de Terre des hommes, Jean Paulhan et Roger Caillois...

[...] Rendons leur place aux essayistes dans toute littérature digne du nom, et surtout d'expression française ${ }^{44}$.

L'essayiste incarne donc ces vertus de penseur actif qui sont le propre du poète, de celui qui façonne. Ainsi, parmi les rares descriptions que Rougemont ait données de la figure de l'écrivain telle qu'il la souhaite, il nous le présente comme le «prophète, que certains nomment l'utopiste $»^{45}$, qui révèle son époque, la conteste et la transforme par sa parole fondatrice. Son œuvre consiste en :

[...] la donation d'une mesure, la création de formes, de concepts, et l'expression de modes de sentir qui donnent « un sens plus pur aux mots de la tribu », et instaurent ou restaurent la communauté [...].

Paroles de poète, paroles de prophète, c'est autant dire de fondateur.

Ce que l'écrivain doit au monde et à l'événement, c'est de les créer.

Et ce qu'il faut attendre du meilleur écrivain, c'est qu'il fasse converger dans son œuvre le sentiment baudelairien de son époque, la révolte contre elle de tout homme qui se veut tel, et l'annonce admirable d'un monde équilibré - dans son mouvement ${ }^{46}$.

On disait plus haut que l'écrivain ne s'accomplit pas différemment de la personne : on voit ici que la portée de sa vocation revêt une importance particulière. En témoignant de ce que l'action par la vocation a de rénovateur, il devient l'exemple manifeste de ce devoir humain.

L'analogie avec le prophète ne nous renvoie toutefois pas au prophète-devin, tel que nous l'avait présenté Paul Bénichou pour l'époque romantique ${ }^{47}$. Il ressemble davantage à l'homme qui «n'a pas de biographie. Il se lève et tombe avec sa mission ", comme le disait Karl Barth ${ }^{48}$ (ou, en termes personnalistes, celui qui incarne purement sa vocation). Mais les deux figures ne se recoupent pas à la perfection; le poète peut être prophète, mais reste avant tout un homme au monde, avec ses contingences. Cette très haute mission de donner à l'humain des catégories pour sa pensée au travers d'une parole plénière, le poète ne la réalisera pas tous les jours : l'événement prophétique, sans doute, ne l'accompagnera que par instants. Figure quasi mythique, comme l'utopiste qui concrétiserait un mode de vie foncièrement différent, cette condition n'est revêtue qu'un moment - le temps de l'acte amenant au monde ce qui jusque-là lui manquait.

L'écrivain, le «littéraire », serait donc pris à tort comme une figure subsidiaire et accommodante à l'ordre du monde : il est celui - et probablement, croyons-nous, tel que Rougemont s'est rêvé - celui dont la parole quasi divine, en ce qu'elle coïncide avec sa vocation, sait inspirer le sens, le mouvement, l'engagement nécessaires à ses semblables. Qu'il faille un acte de foi pour suivre pareille conception, c'est certain : 
mais le risque, pour reprendre un mot qui lui était cher, c'est notre option permanente face à la fatalité.

\section{BIBLIOGRAPHIE}

ACKERMANN, Bruno, Denis de Rougemont : une biographie intellectuelle, Genève, Labor et Fides, 1996,2 vols.

BÉNICHOU, Paul, Le sacre de l'écrivain : 1750-1830 : essai sur l'avènement d'un pouvoir spirituel laïque dans la France moderne, Paris, J. Corti, 1973.

BONDI, Damiano, La persona e l'Occidente : filosofia, religione e politica in Denis de Rougemont, Milano, Mimesis, 2014.

GROETHUYSEN, Bernard, « Sur l'antichristianisme de Nietzsche », in Autres portraits, Paris, Gallimard, 1995, p. 141-150.

MOUNIER, Emmanuel, Manifeste au service du personnalisme, Paris, Esprit, 1936.

OUSSELIN, Edward, « Rougemont and the Literary Construction of Europe », Dalhousie French Studies, 76 (fall 2006), p. 73-84.

ROSE, Martin, « La naissance du penseur de la “ personne ” ", Revue historique neuchâteloise, 1562 (2019/1-2), p. 27-45.

ROUGEMONT, Denis de, « André Gide ou le style exquis », Foi et Vie, 31 (1931), p. 725-729.

—, «Définition de la personne », Esprit, 27 (décembre 1934), p. 368-382.

—, « Mystère de la vision », Hermès, 4 (mars 1935), p. 42-54.

-, Penser avec les mains, Paris, Albin Michel, 1936

—, Nicolas de Flue, légende dramatique, Neuchâtel, Institut Neuchâtelois, 1939.

—, Mission ou démission de la Suisse, Neuchâtel, À la Baconnière, 1940.

—, «Para qué sirven los escritores ? », Sur, Buenos Aires, 86 (novembre 1941), p. 31-48.

—, Les personnes du drame, Neuchâtel, À la Baconnière, 1946.

—, Politique de la personne, Paris, Je Sers ; Neuchâtel, Delachaux et Niestlé, 1946 (1934).

—, « Théologie et littérature », in Hommage et reconnaissance : recueil de travaux publiés à l'occasion du soixantième anniversaire de Karl Barth, Neuchâtel, Delachaux et Niestlé, 1946, p. 162-167.

—, Doctrine fabuleuse, Neuchâtel, Ides et Calendes, 1948.

—, « Calvin », in Tableau de la littérature française, t. I, De Rutebeuf à Descartes, Paris, Gallimard, 1962, p. 276-281.

-, Journal d'une époque, Paris, Gallimard, 1968.

—, Réponse à l'enquête Pourquoi j'écris, Lausanne, La Gazette Littéraire, 1971, p. 89-91.

—, Responsabilité de l'écrivain dans la société européenne d'aujourd'hui, Zurich, Pro Helvetia, 1973. 
-, « L'Europe des consciences », Entailles, 23 (1986), p. 77-83.

_, Inédits, éd. par Jean Mantzouranis, François Saint-Ouen, Neuchâtel, À la Baconnière, 1988.

STAROBINSKI, Jean, « Personne, masque, visage », in André Reszler, Henry Schwamm (éds), Denis de Rougemont : l'écrivain, l'Européen, Neuchâtel, À la Baconnière, 1976, p. 289-297.

WENGER, Jonathan, « La naissance de l'écrivain », Revue historique neuchâteloise: «Union, Étude». Denis de Rougemont, 156/1-2 (2019), p. 47-62.

\section{NOTES}

1. Cet article reprend et développe une partie d'un mémoire de Master soutenu à l'Université de Neuchâtel en septembre 2016 : L'œuvre de jeunesse de Denis de Rougemont : la formation littéraire de la personne. Nous remercions le professeur Alain Corbellari, qui l'a dirigé avec Daniel Maggetti, d'en accueillir ce fragment.

2. Pour une vision aussi brève que pertinente de ces points, voir E. Ousselin, « Rougemont and the Literary Construction of Europe ».

3. Si l'on trouve quelques éléments biographiques dans B. Ackermann, Denis de Rougemont (sp. chap. II-VIII), le premier ouvrage à situer la perspective théologique de Rougemont est celui de D. Bondi, La persona e l'Occidente ; voir également sa contribution à ce numéro et M. Rose, «La naissance du penseur de la " personne" ".

4. On parlera toujours ici d'un personnalisme propre à Denis de Rougemont : les membres liés de près ou de loin aux différents ensembles de cette mouvance ont toujours refusé d'en établir une orthodoxie qu'ils jugeraient sclérosante. Les différences sont toutefois rarement fondamentales. Chez Rougemont, il s'agit spécialement de concevoir la vocation comme un rapport personnel à Dieu - alors qu'elle tient davantage, chez Emmanuel Mounier par exemple, d'une conscience de soi et de son devoir particulièrement fine (cf. son Manifeste au service du personnalisme, p. $69 \mathrm{sq}$.). Pour la dimension historique du développement du personnalisme, ainsi que quelques notions, cf. B. Ackermann, Denis de Rougemont, p. 299-384.

5. Pour l'importance de la littérature dans la jeunesse de notre auteur, on se permet de renvoyer à notre article : « La naissance de l'écrivain ».

6. Cf. p. ex. Politique de la personne, p. 57 sq.

7. Cf. p. ex. «Définition de la personne », p. 375 sq.

8. Mission ou démission de la Suisse, p. 84-77.

9. Descartes a ainsi «détruit la personne, ou plutôt son lieu naturel, en séparant le corps et l'âme." ("Définition de la personne », p. 380). Ce reproche court dans toute l'œuvre; on le retrouve, entre autres, dans Penser avec les mains, p. 178 sq., ou dans Les personnes du drame, p. 195, à propos de "Descartes le diviseur ». Voir encore sur les abus de la technique comme pensée stérile dont le philosophe serait à l'origine, Inédits, p. 62.

10. Pour ces critiques, voir notamment Penser avec les mains, spécialement p. 164-166 et 190-192 ; Politique de la personne, p. 40-45; et Mission ou démission de la Suisse, p. 90-92.

11. «Définition de la personne ", p. 371.

12. Politique de la personne, p. 22. Dans les citations, c'est toujours Rougemont qui souligne.

13. B. Groethuysen, «Sur l'antichristianisme de Nietzsche » [1926], p. 143.

14. «Définition de la personne », p. 372 sq.

15. Mission ou démission de la Suisse, p. 31 sq.

16. «Définition de la personne ", p. 372.

17. Reprise encore dans Inédits, p. 164 sq. Cette étymologie est aujourd'hui clairement tenue pour fautive, les quantités vocaliques de personare ne permettant pas d'aboutir à persona. Voir ce qu'en 
dit Jean Starobinski : "Une étymologie populaire, et sans doute fausse (per-sonare, retentir au travers) fait de la persona le lieu du passage et l'instrument de l'amplification d'une voix venue d'ailleurs [...]» (« Personne, masque, visage », p. 289).

18. Selon l'acception moderne du terme qu'avait imposée Benda avec La trahison des clercs dès 1927. S'opposant directement au désengagement qui y était préconisé, Rougemont recourt souvent lui-même à ce terme.

19. Outre la disposition de l'action, voir le dispositif allégorique de la mise en scène que commente Rougemont dans son Journal d'une époque, p. 368.

20. Penser avec les mains, p. 207.

21. Pour celles qui nous intéressent, cf. Ibid., p. 215-231.

22. Ibid., p. 215 sq.

23. Ibid., p.

24. Mission ou démission de la Suisse, p. 66.

25. Penser avec les mains, p. 224.

26. "À quoi servent les écrivains ?", tapuscrit conservé dans le Fonds Denis de Rougemont, à la Bibliothèque publique et universitaire de Neuchâtel, ID 430, f. 18 du tapuscrit. Cette conférence, qui nous intéresse notamment sur les questions de l'ascétisme et de l'autorité, a été donnée en Argentine et publiée en espagnol, sous le titre « Para qué sirven los escritores?».

27. Penser avec les mains, p. 227.

28. « Mystère de la vision », p. 45.

29. Penser avec les mains, p. 227.

30. Réponse à l'enquête Pourquoi j'écris, p. 91.

31. Penser avec les mains, p. 230 ; voir aussi p. 244. Pour une esquisse des rapports de Gide et de Rougemont, voir J. Wenger, « La naissance de l'écrivain ».

32. Les personnes du drame, p. 156.

33. « André Gide ou le style exquis ».

34. Les personnes du drame, p. 157 sq. pour ces deux dernières citations. La déformation en question provient de la croyance erronée que l'écriture pourrait être observation neutre. Or écrire, c'est réitérer, donc renforcer l'observation faite. Contre Gide, elle ne saurait être un acte gratuit.

35. Rappelons la présence, dans la liste de ces huit vertus, de la violence comme capacité d'atteindre, comme impact sur un état. Voir Penser avec les mains, p. 207 sq. et p. 152 sq.

36. "Théologie et littérature ", p. 166 sq.; comme il le remarquera de Calvin : « Toutes les vertus de son style découlent de cette tension instaurée par sa vocation » («Calvin », p. 279).

37. Les personnes du drame, p. 12. Nous soulignons.

38. On reprend ici une expression plus vastement employée dans Politique de la personne, p. 207, extrapolant encore Calvin.

39. Une vision téléologique et humainement déductible de l'histoire, au sens d'un déroulement strictement linéaire et non orienté par une fin, serait inacceptable à Rougemont, puisque découlant des excès du rationalisme.

40. «Si toute présence est l'événement de l'éternel dans le temps, par le moyen de l'homme, si l'homme n'est vraiment homme que dans l'acte qui fonde sa qualité incomparable de sujet, [...] on peut dire que la personne est l'impensable incarnation de l'éternité dans le temps » (« Définition de la personne », p. 375).

41. Les personnes du drame, p. 16.

42. Au sens évoqué plus haut.

43. Les personnes du drame, p. 19.

44. "L'Europe des consciences », p. 77. Au vu des pages précédentes, la convocation de Descartes peut surprendre; on rappelle qu'il faisait de lui davantage le symbole de la pensée abstraite qu'un adversaire personnel. Pour les nuances, voir Doctrine fabuleuse, p. 37-42. 
45. Responsabilité de l'écrivain dans la société européenne d'aujourd'hui, p. 15.

46. «L'écrivain et l'événement », p. 189 sq.

47. Voir évidemment Le sacre de l'écrivain.

48. Repris de Parole de Dieu et parole humaine, que Rougemont cite deux fois, dans Penser avec les mains, p. 50 et dans Les personnes du drame, p. 74.

\section{RÉSUMÉS}

En examinant la théorie personnaliste de Denis de Rougemont, en analysant les métaphores privilégiées (d'ordres dramaturgique et rhétorique), enfin en précisant dans sa pensée le caractère existentiel du discours et du langage, on tente ici de démontrer la dimension à la fois fondatrice et originale de la littérature dans l'œuvre de l'écrivain.

\section{AUTEUR}

\section{JONATHAN WENGER}

Université de Genève 